\title{
Activation of NMDA Receptors in the Suprachiasmatic Nucleus Produces Light-Like Phase Shifts of the Circadian Clock In Vivo
}

\author{
Eric M. Mintz, ${ }^{1}$ Cherie L. Marvel, ${ }^{1}$ Charles F. Gillespie, ${ }^{1}$ Kristina M. Price, ${ }^{1}$ and H. Elliott Albers ${ }^{1,2}$ \\ Laboratory of Neuroendocrinology and Behavior, Departments of ${ }^{1}$ Biology and ${ }^{2}$ Psychology, Georgia State University, \\ Atlanta, Georgia 30303
}

Although there is substantial evidence that glutamate mimics the effects of light on the mammalian circadian clock in vitro, it has been reported that microinjection of glutamate into the suprachiasmatic nucleus of the hypothalamus (SCN) region in vivo does not result in a pattern of phase shifts that mimic those caused by light pulses. The present study was designed to test the hypothesis that microinjection of NMDA into the SCN would induce light-like phase shifts of the circadian clock through activation of the NMDA receptor. Hamsters housed in constant darkness received microinjections of NMDA through guide cannulas aimed at the SCN region at various times throughout the circadian cycle. Wheel running was monitored as a measure of circadian phase. Microinjection of NMDA resulted in circadian phase shifts, the size and direction of which were dependent on the time of injection. The resulting phase-response curve closely resembled that of light. The circadian response showed a clear dose-dependence at circadian time (CT) 13.5 but not at CT19. Both phase delays and advances induced by NMDA were blocked by coinjection of the NMDA antagonist 2-amino5-phosphopentanoic acid but were slightly attenuated by the non-NMDA antagonist 6-nitro-7-sulfamoylbenzo[f]quinoxaline2,3-dione disodium. The ability of NMDA to induce phase shifts was not altered by coinjection with tetrodotoxin. These data are consistent with the hypothesis that activation of NMDA receptors is a critical step in the transmission of photic information to the SCN.

Key words: circadian rhythm; glutamate; NMDA; Syrian hamster; wheel running; suprachiasmatic nucleus
The suprachiasmatic nucleus of the hypothalamus (SCN) is the location of the primary circadian clock in mammals. The SCN is responsible for driving a variety of daily rhythms in behavior and physiology. Under constant environmental conditions [i.e., constant darkness (DD)] the SCN drives rhythms that are close to but not exactly $24 \mathrm{hr}$. These rhythms are normally synchronized to the $24 \mathrm{hr}$ day through the process of entrainment (the resetting of the clock each day to synchronize the timing of the clock with environmental time cues). The most important environmental cue for entrainment is light.

Photic information reaches the SCN via several pathways. There is a direct projection of retinal ganglion cells to the SCN, forming a retinohypothalamic tract (RHT) (Moore and Lenn, 1972; Pickard, 1982; Johnson et al., 1988b; Levine et al., 1991). There are also multisynaptic pathways from the retinas to the SCN, including projections from the intergeniculate leaflet of the thalamus (Moore and Card, 1985, 1994; Card and Moore, 1989, 1991; Morin et al., 1992; Morin, 1994). However, only ablation of the RHT (Johnson et al., 1988a) or blinding (Richter, 1968) results in the loss of the ability of light to entrain circadian rhythmicity. Several neurochemical signals have been localized in the RHT, including glutamate, aspartate, pituitary adenylate cyclase-activating peptide, and substance P (van den Pol and Tsujimoto, 1985; Albers et al., 1992; Piggins et al., 1996; Hannibal et al., 1997). These neurochemicals are therefore the most likely candidates for transmitting photic information to the SCN.

Received Jan. 22, 1999; revised March 25, 1999; accepted March 30, 1999.

This research was supported by National Institutes of Health Grants NS34586 and MH58789 to H.E.A. and NS09927 to E.M.M.

Correspondence should be addressed to Eric M. Mintz, Laboratory of Neuroendocrinology and Behavior, Department of Biology, Georgia State University, Atlanta, GA 30303.

Copyright (C) 1999 Society for Neuroscience $\quad 0270-6474 / 99 / 195124-07 \$ 05.00 / 0$
Glutamate has been localized in RHT terminals in the SCN (van den Pol, 1991; Castel et al., 1993; De Vries et al., 1993; Gannon and Rea, 1993), and ${ }^{3} \mathrm{H}$-glutamate is released in the SCN after optic nerve stimulation (Liou et al., 1986). Application of non-NMDA antagonists blocks excitatory postsynaptic potentials in SCN cells after stimulation of the optic nerves (Cahill and Menaker, 1989). When applied to the hypothalamic slice preparation, glutamate (Ding et al., 1994) or NMDA (Ding et al., 1994; Shibata et al., 1994) induces phase shifts in the rhythm of neuronal firing rate in a pattern that mimics that of light, and microinjection of NMDA or non-NMDA antagonists into the SCN region blocks the phase-shifting effects of light in hamsters (Colwell and Menaker, 1992). These data suggest that glutamate plays a role in transmitting photic information to the circadian clock.

A previous study examined the phase-shifting effects of microinjection of glutamate into the SCN region (Meijer et al., 1988). Microinjection of glutamate into the $\mathrm{SCN}$ region induced phase advances during the subjective day and no significant shifts during subjective night. This pattern strongly resembles the phaseshifting effects of dark pulses (Boulos and Rusak, 1982; Ellis et al., 1982). This result, therefore, did not support the hypothesis that glutamate transmits photic information to the SCN. Recently, however, we demonstrated that microinjection of NMDA into the $\mathrm{SCN}$ region could produce both phase delays and phase advances of the circadian locomotor activity rhythm in hamsters that were similar to those produced by a brief light pulse (Mintz and Albers, 1997). The reason for the discrepancies between the two studies remain to be resolved, but our results do support the hypothesis that the release of glutamate in the $\mathrm{SCN}$ is a primary mechanism by which photic information is transmitted to the circadian clock.

The present study was designed to test the hypotheses that NMDA exerts its effects on circadian rhythms through activation 
of NMDA-type glutamate receptors, that these effects mimic the phase-shifting effects of light in vivo and glutamate in vitro, and that exogenously applied NMDA was acting directly on clock cells in the SCN.

\section{MATERIALS AND METHODS}

Adult male Syrian hamsters (Mesocricetus auratus) were purchased from Charles River Laboratories (Wilmington, MA). Hamsters were individually housed in Plexiglas cages $(20 \times 40 \times 20 \mathrm{~cm})$ equipped with running wheels (diameter, $16 \mathrm{~cm}$ ). Food and water were available ad libitum. Each wheel revolution activated a microswitch on the outside of the cage, which was monitored continuously by a 386 microcomputer using DataCol 3 software (Mini-Mitter, Sunriver, OR).

Each hamster (130-160 gm) was deeply anesthetized with a cocktail comprised of ketamine $(120 \mathrm{mg} / \mathrm{kg})$, xylazine $(25 \mathrm{mg} / \mathrm{kg})$, and acepromazine $(2 \mathrm{mg} / \mathrm{kg}$ ) (Butler Co., Atlanta, GA) and was stereotaxically implanted with an 26 gauge guide cannula (11 $\mathrm{mm}$ total length) aimed at the SCN. Stereotaxic coordinates were $0.8 \mathrm{~mm}$ anterior and 1.7 $\mathrm{mm}$ lateral to bregma, and with a 32 gauge injection needle inserted into the cannula, the final depth was $7.2 \mathrm{~mm}$ below dura. The cannulas were implanted at a $10^{\circ}$ angle toward the midline, and the skull was leveled before implantation using bregma and lambda as reference points. After surgery, hamsters were housed in DD and were allowed to establish stable free-running activity rhythms. After 7-10 d of stable running, microinjections were given with a $16 \mathrm{~mm}, 32$ gauge needle attached by polyethylene tubing to a $1 \mu$ l Hamilton syringe. Injections were given in dim red illumination to hamsters that were gently restrained by hand. After each injection, the needle was left in place for at least $15 \mathrm{sec}$. A minimum of $9 \mathrm{~d}$ separated multiple treatments given to any individual animal. The experiments conducted were as follows.

Phase-response curve. Animals received a $200 \mathrm{nl}$ microinjection of 10 mM NMDA [in 0.9\% saline (SAL); Research Biochemicals, Natick MA] at times throughout the circadian day. Each animal received up to three injections.

Dose-response curves. Animals received $200 \mathrm{nl}$ microinjections of 10, 5, 1, or 0.5 mM NMDA or saline at circadian time (CT) 13.5 or CT19CT20 to generate dose-response curves for NMDA microinjection at these time points. Each animal received up to three injections.

Antagonists. Animals received $200 \mathrm{nl}$ microinjections of $10 \mathrm{~mm}$ NMDA, $10 \mathrm{~mm}$ NMDA plus $10 \mathrm{~mm}$ 2-amino-5-phosphopentanoic acid (AP-5), $10 \mathrm{~mm}$ NMDA plus $10 \mathrm{~mm}$ 6-nitro-7-sulfamoylbenzo[f]quinoxaline-2,3-dione disodium (NBQX), $10 \mathrm{~mm}$ AP-5, $10 \mathrm{~mm}$ NBQX (all from Research Biochemicals), or saline at CT13.5 or CT19-CT20. The NMDA plus AP-5 and NMDA plus NBQX treatments were delivered as a drug cocktail.

Tetrodotoxin. Animals received $200 \mathrm{nl}$ microinjections of $4 \mu \mathrm{M}$ tetrodotoxin (TTX) (Sigma, St. Louis, MO) or saline, followed by either a 15 min light pulse (20 lux) or a microinjection of $10 \mathrm{mM} \mathrm{NMDA}$ at CT13.5.

After completion of the experiment, hamsters were given an overdose of sodium pentobarbital and microinjected with $200 \mathrm{nl}$ of India ink, and their brains were removed and stored in 10\% formalin. Tissue sections (100 $\mu \mathrm{m}$ thickness) were sliced on a vibratome, and injection sites were verified. Only animals with injection sites that did not penetrate the third ventricle and were within $300 \mu \mathrm{m}$ of the SCN were used in the study.

Phase shifts in the circadian activity rhythm were quantified using the linear regression method of Daan and Pittendrigh (1976). This method estimates the circadian period and phase by fitting regression lines through the daily onsets of activity for $6-7 \mathrm{~d}$ before and 8-10 d after microinjection. The first three activity onsets after treatment were not included in the second regression line to avoid the influence of transient or unstable onsets. If the SE of either regression line was greater than 15 min, the trial was excluded from further analysis. Phase shifts were calculated from the difference between the onset of activity predicted by the first line and the actual line observed after drug treatment. One-way ANOVA and Newman-Keuls post hoc pairwise comparisons were used to evaluate statistical differences between experimental and control groups, and significance was ascribed at $p<0.05$.

\section{RESULTS}

\section{Phase-response curve}

The phase shifts produced by microinjection of NMDA result in a phase-response curve (PRC) that is similar to that produced by brief pulses of light (Fig. 1). In the early part of the subjective

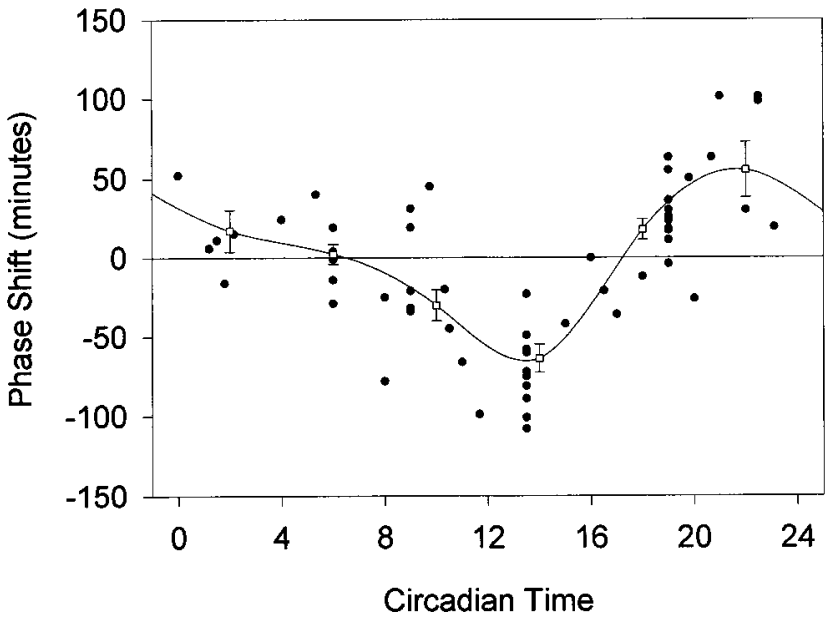

Figure 1. Phase shifts in minutes induced by microinjection of NMDA into the SCN region, plotted as a function of the circadian time of the injection. Negative values represent phase delays, and positive values represent phase advances. Open squares represent means \pm SE for values grouped into $4 \mathrm{hr}$ bins.

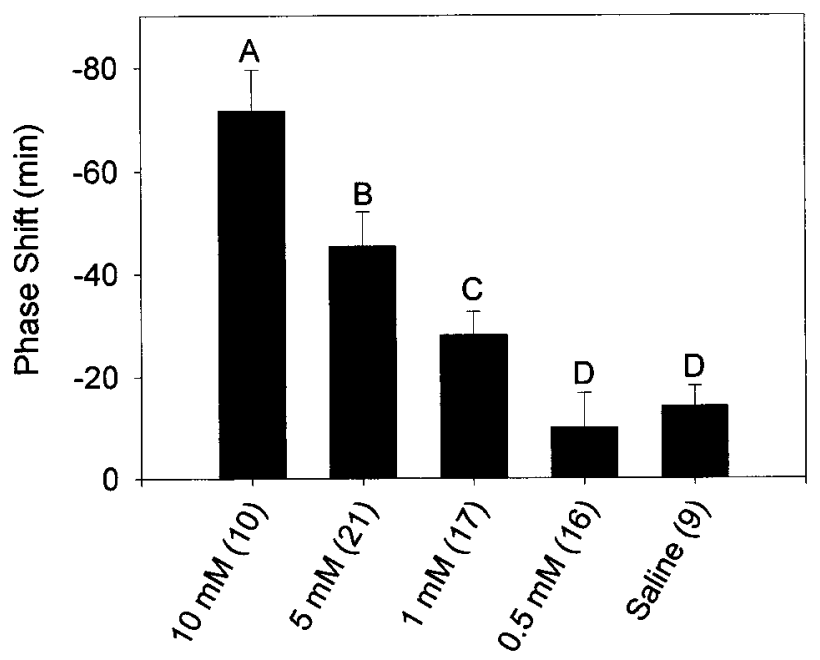

Figure 2. Mean \pm SEM phase shift in minutes induced by microinjection of NMDA into the SCN region at CT13.5. Sample sizes are denoted in parentheses. Negative values represent phase delays. Letters denote significant differences between groups $(p<0.05)$ as follows: $A$, differs from all other groups; $B$, differs from 10,5 , and $0.5 \mathrm{~mm}$ groups; $C$, differs from 10 $\mathrm{mm}$ group; $D$, differs from 10 and $5 \mathrm{~mm}$ groups.

night, microinjection of NMDA into the SCN produces phase delays of the circadian locomotor activity rhythm, whereas microinjection of NMDA in the latter part of the subjective night results in phase advances. The phase delay portion of the curve extends into the late subjective day, appearing to begin at approximately CT9. The amplitude of the phase delay portion of the curve appears to be slightly greater than in the phase advance portion of the curve. The largest phase delay produced was 108 min at CT13.5, and the largest phase advances were $101 \mathrm{~min}$ at CT21 and CT22.5.

\section{Dose-response curves}

At CT13.5, the phase-shifting effects of NMDA had a clear dose-dependence (Fig. 2). A concentration of $1 \mathrm{~mm}$ was sufficient to produce phase delays that were significantly greater than saline injections. This was not the case at CT19-CT20, where all doses 


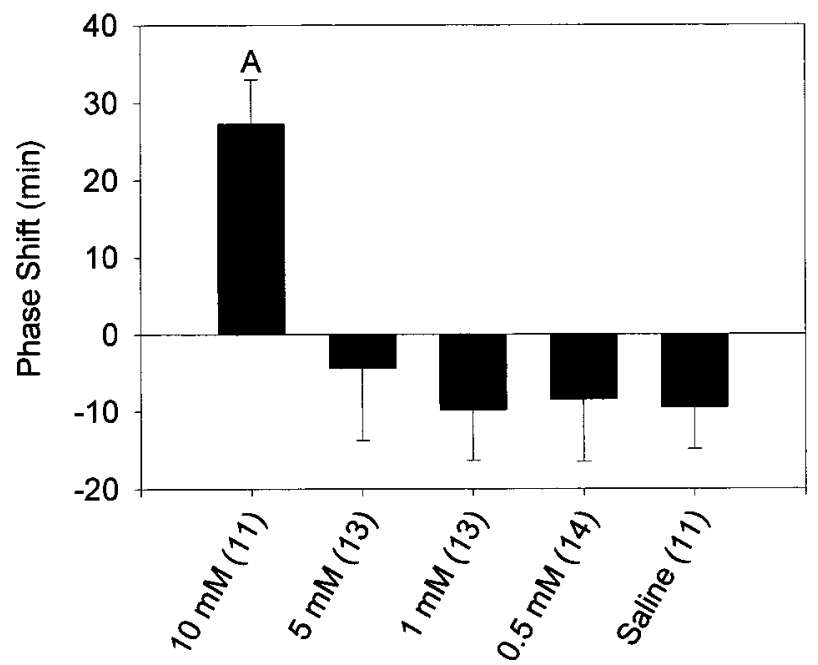

Figure 3. Mean \pm SEM phase shift in minutes induced by microinjection of NMDA into the SCN region at CT19-CT20. Sample sizes are denoted in parentheses. Negative values represent phase delays, and positive values represent phase advances. $A$, Significantly differs from all other groups $(p<0.05)$.

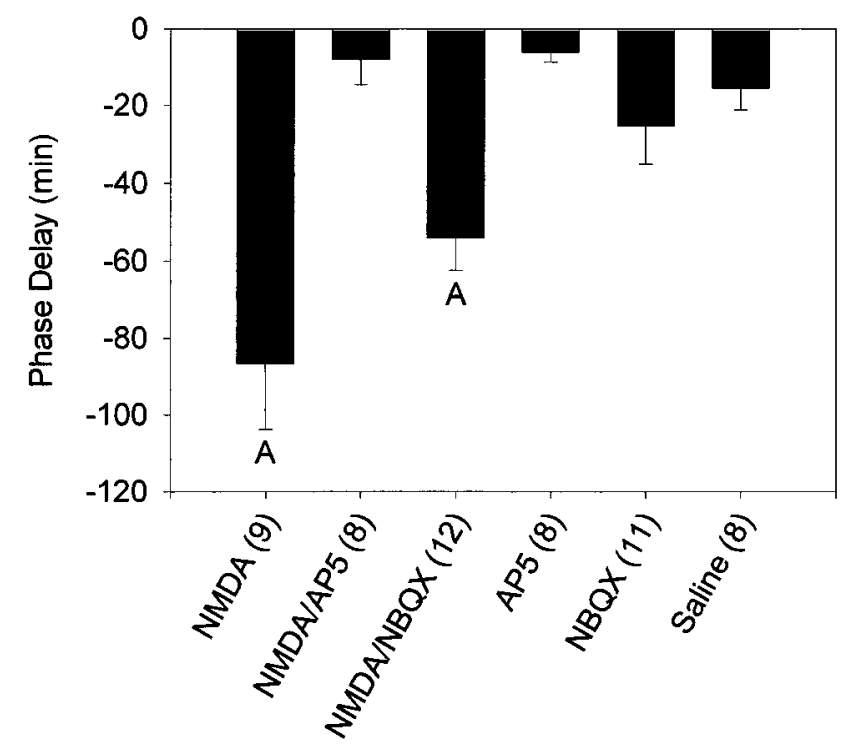

Figure 4. Mean \pm SEM phase shift in minutes induced by microinjection of AP-5 and NBQX and their effects on NMDA-induced phase shifts at CT13.5. Sample sizes are denoted in parentheses. Negative values represent phase delays. $A$, Significantly differs from all other groups $(p<0.05)$.

below $10 \mathrm{~mm}$ failed to induce significant phase shifts (Fig. 3). The dose-response curves were not extended to concentrations higher than $10 \mathrm{~mm}$ because of concerns about inducing excitotoxic damage to the SCN region.

\section{Specificity to NMDA receptor}

At CT13.5, coadministration of the NMDA antagonist AP-5 completely blocked the phase delays produced by NMDA, whereas the non-NMDA antagonist NBQX slightly attenuated the phase shift (Figs. 4-6). Both the NMDA and NMDA-NBQX groups were significantly different from all other groups, including each other. Neither AP-5 nor NBQX produced phase shifts on their own that were significantly different from saline injections. At CT19-CT20, AP-5 again blocked NMDA-induced

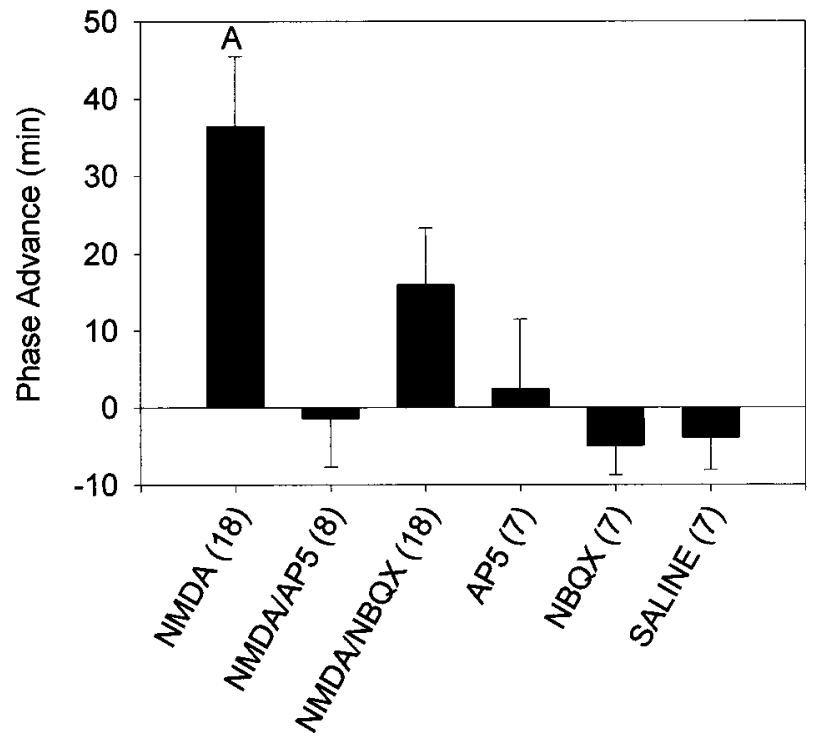

Figure 5. Mean \pm SEM phase shift in minutes induced by microinjection of AP-5 and NBQX and their effects on NMDA-induced phase shifts at CT13.5. Sample sizes are denoted in parentheses. Negative values represent phase delays, and positive values represent phase advances. $A$, Significantly differs from all other groups, except NMDA-NBQX $(p<0.05)$.

phase shifts, but NMDA combined with NBQX resulted in phase shifts that were intermediate between NMDA-SAL and SAL without being significantly different from either (Figs. 5, 7). Phase shifts produced after NMDA-NBQX were not significantly different from any other group, whereas NMDA alone was significantly different from all other groups.

\section{Dependence on synaptic transmission}

TTX injected into the SCN region immediately before a $15 \mathrm{~min}$ light pulse at CT13.5 significantly reduced the resulting phase delay (Fig. 8). However, TTX injected immediately before NMDA injection did not alter the phase-shifting effects of NMDA.

\section{DISCUSSION}

This study provides critical evidence in support of the hypothesis that activation of NMDA receptors is a sufficient and necessary step in the transduction of photic information to the circadian clock located in the SCN. Previous research had demonstrated that microinjection of either NMDA antagonists or non-NMDA antagonists into the SCN region could block the phase-shifting effects of light (Colwell and Menaker, 1992) and that application of glutamate to the hypothalamic slice preparation could phase shift the SCN neuronal firing rhythm in a pattern similar to that produced by light pulses in vivo (Ding et al., 1994). Recently, we demonstrated that microinjection of NMDA into the SCN region was capable of producing both phase delays and phase advances of the circadian locomotor activity rhythm (Mintz and Albers, 1997). This paper extends that finding to demonstrate that direct application of NMDA to the SCN in vivo produces a light-like phase-response curve, that this effect is specific to NMDA receptors, and that NMDA probably exerts effects directly on pacemaker cells and/or cells nonsynaptically coupled to pacemaker cells.

The PRC produced by microinjection of NMDA into the SCN region is qualitatively similar to that produced by brief pulses of light (DeCoursey, 1964; Daan and Pittendrigh, 1976). However, 


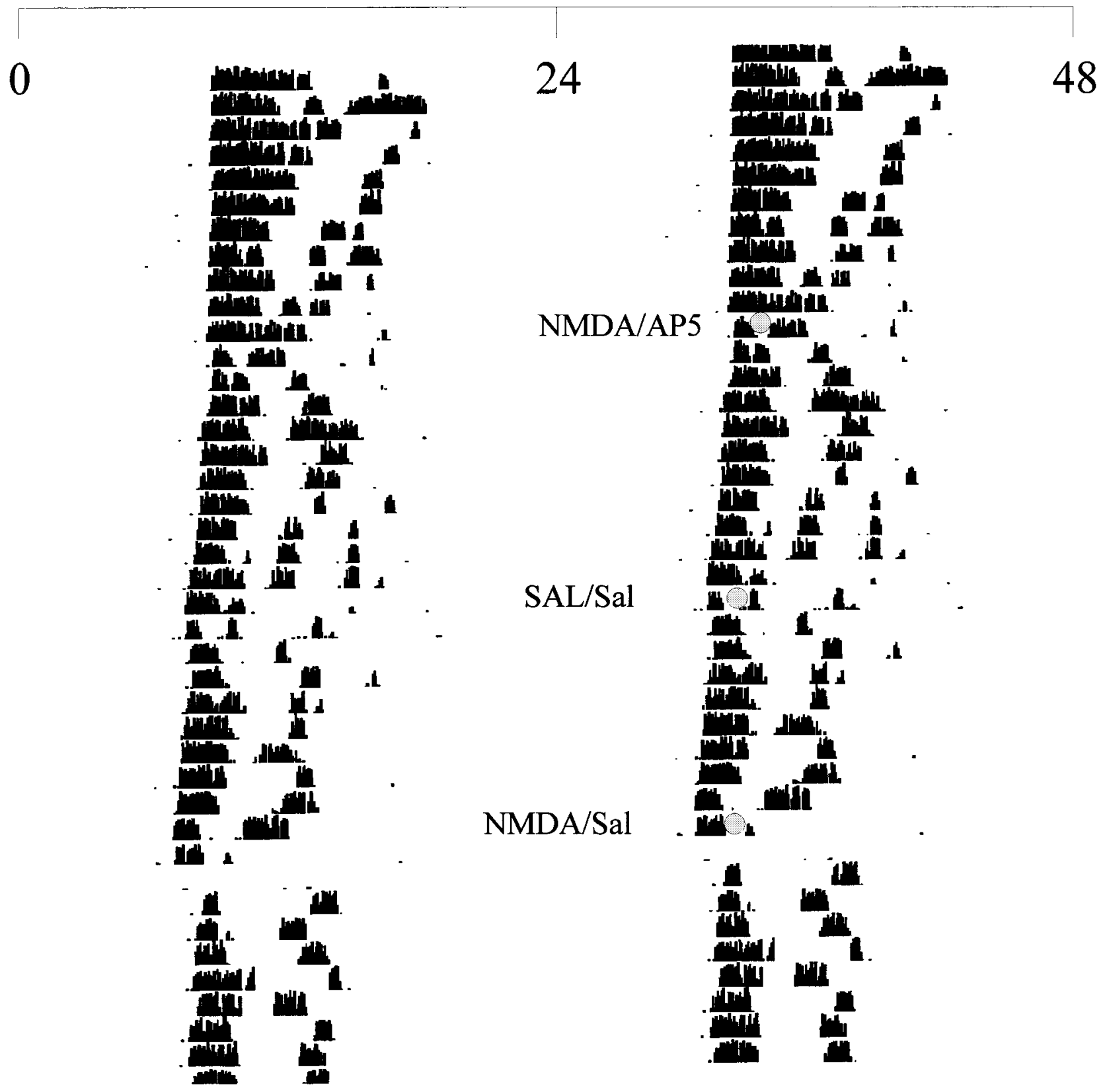

Figure 6. Representative double-plotted actogram illustrating the wheel-running activity in constant darkness of a hamster receiving injections at CT13.5. Each line represents one $48 \mathrm{hr}$ period, and successive days are shown from top to bottom. Injections were given at the times marked by shaded circles using dim red illumination.

the curve appears to deviate from a light-induced PRC in two ways. First, the delay portion of the curve begins during the late subjective day, at approximately CT9, rather than at the onset of subjective night. Although the reasons for this difference are unknown, one explanation could lie in the effects of GABA on light-induced phase shifts of the circadian clock. SCN cell firing rate is higher during the day (Green and Gillette, 1982), and GABAergic cells are distributed throughout the SCN (Card and Moore, 1984; van den Pol and Tsujimoto, 1985; Okamura et al., 1989; Moore and Speh, 1993). One would expect, therefore, that local GABA release in the SCN may be higher during the day than at night. Furthermore, there is evidence that $\mathrm{GABA}_{\mathrm{B}}$ receptor activation inhibits the release of glutamate in the SCN (Jiang et al., 1995). Microinjection of the $\mathrm{GABA}_{\mathrm{B}}$ agonist baclofen into the SCN region significantly reduces light-induced phase delays, whereas the GABA $_{\mathrm{B}}$ antagonist CGP-35348 significantly enhances light-induced phase delays (Gillespie et al., 1997). Therefore, high concentrations of GABA in the late subjective day may normally inhibit phase-delaying effects of light but would not block the phase-shifting effects of NMDA in late afternoon, because this is likely to be a postsynaptic effect.

Another aspect of the NMDA PRC is that the amplitude of phase advances appears to be equal to or slightly lower than phase delays, whereas in hamsters light-induced phase advances are generally larger than phase delays for a given stimulus at the maximal points of the curve. This could simply be because of the characteristics of the NMDA microinjection as a stimulus. The NMDA is given as a bolus injection, whereas light pulse PRCs are 


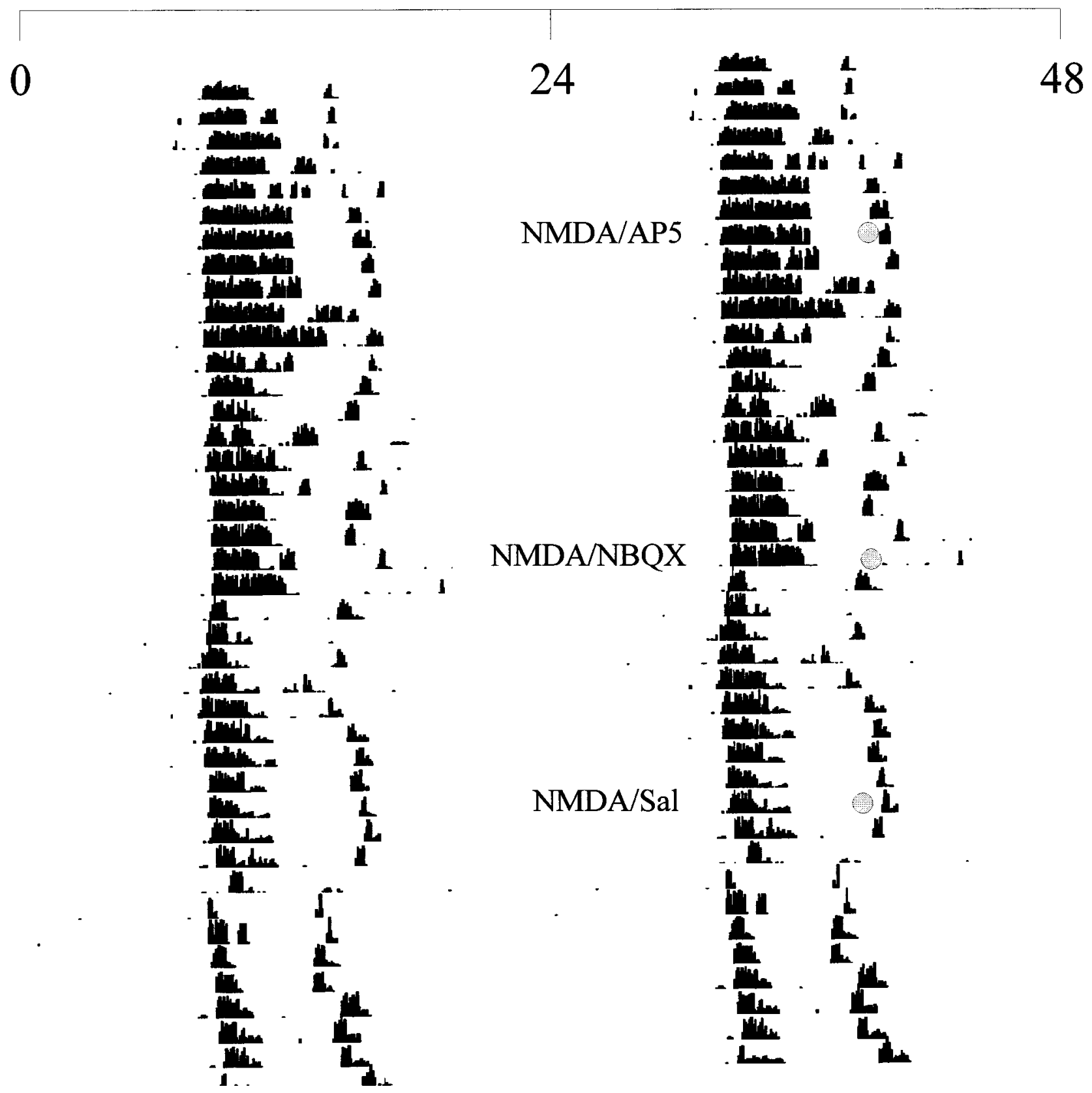

Figure 7. Representative double-plotted actogram illustrating the wheel-running activity in constant darkness of a hamster receiving injections at CT19. Each line represents one $48 \mathrm{hr}$ period, and successive days are shown from top to bottom. Injections were given at the times marked by shaded circles using dim red illumination.

generally made with a 10 min or longer pulse of light, and the clearance time of the exogenous NMDA may differ at the two time points. Another possibility is that a cofactor is released during the phase advance portion of the PRC that enhances glutamatergic stimulation and that this cofactor is not present in sufficient quantities at CT19-CT20 when no light pulse is presented. The most likely explanation, however, results from the fact that saline injections resulted in an $\sim 10$ min phase delay in both the early and late night (Figs. 2, 3). If this effect were additive with the phase-shifting effects of NMDA, then the magnitude of the phase shifts attributable to NMDA would be reduced by $10 \mathrm{~min}$ at CT13.5 and increased by $10 \mathrm{~min}$ at CT19CT20. This would result in a ratio of amplitudes between the phase delay and phase advance parts of the phase-response curve that more closely resembles that produced by brief light pulses.

Despite these differences, the PRC from microinjection of NMDA is markedly different from that produced by microinjection of glutamate into the SCN (Meijer et al., 1988). However, there were a number of methodological differences between the two studies, apart from the use of NMDA in place of glutamate. In particular, the glutamate injections were anesthetized briefly before the injection, which could have altered the effects of glutamate on circadian rhythms. A variety of anesthetics have been demonstrated to block the phase-shifting effects of light (Colwell et al., 1993b).

Curiously, at CT13.5, the phase-shifting effects of NMDA 


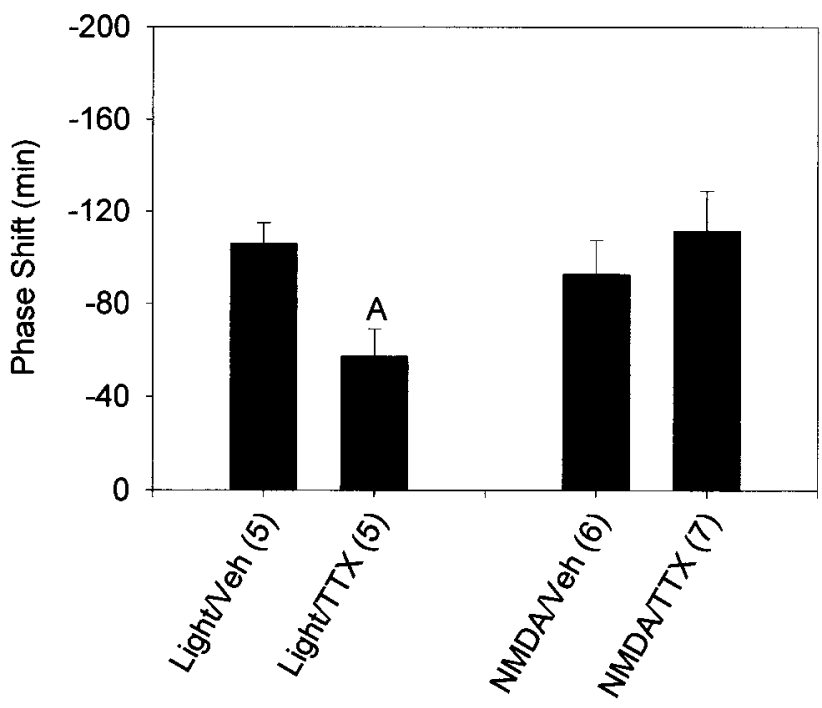

Figure 8. Mean \pm SEM phase shift in minutes induced by a 15 min light pulse or a $10 \mathrm{~mm}$ microinjection of NMDA when preceded by microinjection of TTX or vehicle at CT13.5. Sample sizes are denoted in parentheses. Negative values represent phase delays. $A$, Significantly differs from light with vehicle injection $(p<0.05)$.

microinjections show a clear dose-dependence, whereas at CT19CT20, all doses lower than $10 \mathrm{~mm}$ failed to produce significant phase shifts. One possibility is that the SCN is less sensitive to NMDA during the late subjective night and that the doseresponse curve is fairly steep, with significant effects beginning somewhere between 5 and $10 \mathrm{~mm}$. It is also possible that CT19CT20 does not represent the time of maximal phase advances and that a better response to lower doses of NMDA would occur at a later circadian time. Some larger phase advances were seen after microinjection of NMDA at approximately CT22.

Results from a $10 \mathrm{~mm}$ injection of NMDA could potentially represent a pharmacological effect as a result of the high concentration injected. However, estimates for the concentration of glutamate in synapses have gone as high as $15 \mathrm{~mm}$ (Erecinska and Silver, 1990). The previous study, which used SCN microinjections of glutamate at a concentration of $1 \mathrm{~mm}$, found a phaseresponse curve that had phase advances at approximately CT6 and little or no phase shifts at other times of the cycle, which is very different from that seen in the present study (Meijer et al., 1988). It is possible that lower doses of NMDA would have different effects. In the present study, NMDA microinjections were clearly blocked by coadministration of the NMDA antagonist AP-5 and slightly attenuated by the non-NMDA antagonist NBQX. These two antagonists did not induce phase shifts when injected alone. Because activation of non-NMDA receptors is usually necessary for the opening of the NMDA receptor ion channel, it is not surprising that NBQX attenuated the response to NMDA. However, we cannot rule out the possibility that a portion of the phase-shifting response to NMDA is a result of activation of non-NMDA receptors by NMDA. Light-induced phase shifts are blocked by intraventricular administration of either NMDA or non-NMDA antagonists (Colwell and Menaker, 1992). Nevertheless, the results are consistent with the idea that the phase-shifting effects of NMDA are mediated primarily by NMDA receptors and not by some other pharmacological effect.

There is significant supporting evidence that the level of glutamatergic stimulation influences most if not all phase shifts of the circadian clock. Infusion of NMDA and non-NMDA antagonists into the third ventricle blocks the phase-shifting effects of carbachol, a cholinergic agonist (Colwell et al., 1993a). Glutamate blocks the phase-shifting effects of Neuropeptide $\mathrm{Y}$ in vitro (Biello et al., 1997), and glutamate antagonists block the phase-shifting effects of histamine in vitro (Meyer et al., 1998). Taken with the results of the present study, these data suggest that glutamate acts not only to induce phase shifts of the circadian clock but also to modulate other phase-shifting stimuli.

Finally, we examined the hypothesis that NMDA was acting directly on clock cells. Chronic infusion of TTX into the SCN region blocks the phase-shifting effects of light (Schwartz et al., 1987), and in the present study, we demonstrate that a microinjection of TTX into the SCN region immediately before a light pulse attenuates the light-induced phase shift. However, TTX fails to alter the phase-shifting effects of NMDA microinjections. This suggests that sodium-dependent action potentials are not necessary for the transmission of the NMDA signal to clock cells and therefore that NMDA acts directly on clock cells or on cells that are nonsynaptically coupled to clock cells.

\section{REFERENCES}

Albers HE, Liou SY, Stopa EG, Zoeller RT (1992) Neurotransmitter colocalization and circadian rhythms. In: Progress in brain research (Joosse J, Buijs RM, Tilders FJH, eds), pp 289-307. Amsterdam: Elsevier.

Biello SM, Golombek DA, Harrington ME (1997) Neuropeptide Y and glutamate block each other's phase shifts in the suprachiasmatic nucleus in vitro. Neuroscience 77:1049-1057.

Boulos Z, Rusak B (1982) Circadian phase response curves for dark pulses in the hamster. J Comp Physiol 146:411-417.

Cahill GM, Menaker M (1989) Effects of excitatory amino acid receptor antagonists and agonists on suprachiasmatic nucleus responses to retinohypothalamic tract volleys. Brain Res 479:76-82.

Card JP, Moore RY (1984) The suprachiasmatic nucleus of the golden hamster: immunohistochemical analysis of cell and fiber distribution. Neuroscience 13:415-431.

Card JP, Moore RY (1989) Organization of lateral geniculatehypothalamic connections in the rat. J Comp Neurol 284:135-147.

Card JP, Moore RY (1991) The organization of visual circuits influencing the circadian activity of the suprachiasmatic nucleus. In: Suprachiasmatic nucleus: the mind's clock (Klein DC, Moore RY, Reppert SM, eds), pp 51-76. New York: Oxford UP.

Castel M, Belenky M, Cohen S, Ottersen OP, Storm-Mathisen J (1993) Glutamate-like immunoreactivity in retinal terminals of the mouse suprachiasmatic nucleus. Eur J Neurosci 5:368-381.

Colwell CS, Menaker M (1992) NMDA as well as non-NMDA receptor antagonists can prevent the phase shifting effects of light on the circadian system of the golden hamster. J Biol Rhythms 7:125-136.

Colwell CS, Kaufman CM, Menaker M (1993a) Phase shifting mechanisms in the mammalian circadian system: new light on the carbachol paradox. J Neurosci 13:1454-1459.

Colwell CS, Kaufman CM, Menaker M, Ralph MR (1993b) Lightinduced phase shifts and fos expression in the hamster circadian system: the effects of anesthesia. J Biol Rhythms 8:179-188.

Daan S, Pittendrigh CS (1976) A functional analysis of circadian pacemakers in nocturnal rodents. II. The variability of phase response curves. J Comp Physiol 106:253-266.

De Vries MJ, Cardozo BN, van der Want J, de Wolf A, Meijer JH (1993) Glutamate immunoreactivity in terminals of the retinohypothalamic tract of the brown Norwegian rat. Brain Res 612:231-237.

DeCoursey PJ (1964) Function of a light response rhythm in hamsters. J Cell Comp Physiol 63:189-196.

Ding JM, Chen D, Weber ET, Faiman LE, Rea MA, Gillette MU (1994) Resetting the biological clock: nocturnal circadian shifts by glutamate and NO. Science 266:1713-1717.

Ellis GB, McKlveen RE, Turek FW (1982) Dark pulses affect the circadian rhythm of activity in hamsters kept in constant light. Am J Physiol 242:R44-R50.

Erecinska M, Silver IA (1990) Metabolism and role of glutamate in mammalian brain. Prog Neurobiol 35:245-296. 
Gannon RL, Rea MA (1993) Glutamate receptor immunoreactivity in the rat suprachiasmatic nucleus. Brain Res 622:337-342.

Gillespie CF, Mintz EM, Marvel CL, Huhman KL, Albers HE (1997) GABAA and GABAB agonists and antagonists alter the phase shifting effects of light when microinjected into the suprachiasmatic region. Brain Res 759:181-189.

Green DJ, Gillette R (1982) Circadian rhythm of firing rate recorded from single cells in the rat suprachiasmatic brain slice. Brain Res 245:198-200.

Hannibal J, Ding JM, Chen D, Fahrenkrug J, Larsen PJ, Gillette MU, Mikkelsen JD (1997) Pituitary adenylate cyclase-activating peptide (PACAP) in the retinohypothalamic tract: a potential daytime regulator of the biological clock. J Neurosci 17:2637-2644.

Jiang ZG, Allen CN, North RA (1995) Presynaptic inhibition by baclofen of retinohypothalamic excitatory synaptic transmission in rat suprachiasmatic nucleus. Neuroscience 64:813-819.

Johnson RF, Moore RY, Morin LP (1988a) Loss of entrainment and anatomical plasticity after lesions of the hamster retinohypothalamic tract. Brain Res 460:297-313.

Johnson RF, Morin LP, Moore RY (1988b) Retinohypothalamic projections in the hamster and rat demonstrated using cholera toxin. Brain Res 462:301-312.

Levine JD, Weiss ML, Rosenwasser AM, Miselis RR (1991) Retinohypothalamic tract in the female albino rat: a study using horseradish peroxidase conjugated to cholera toxin. J Comp Neurol 306:344-360.

Liou SY, Shibata S, Iwasaki K, Ueki S (1986) Optic nerve stimulationinduced increase of release of ${ }^{3} \mathrm{H}$-glutamate and ${ }^{3} \mathrm{H}$-aspartate but not ${ }^{3} \mathrm{H}$-GABA from the suprachiasmatic nucleus in slices of rat hypothalamus. Brain Res Bull 16:527-531.

Meijer JH, van der Zee EA, Dietz M (1988) Glutamate phase shifts circadian activity rhythms in hamsters. Neurosci Lett 86:177-183.

Meyer JL, Hall AC, Harrington ME (1998) Histamine phase shifts the hamster circadian pacemaker via an NMDA dependent mechanism. J Biol Rhythms 13:288-295.

Mintz EM, Albers HE (1997) Microinjection of NMDA into the SCN region mimics the phase shifting effect of light in hamsters. Brain Res 758:245-249.

Moore RY, Card JP (1985) Visual pathways and the entrainment of circadian rhythms. Ann NY Acad Sci 453:123-133.
Moore RY, Card JP (1994) Intergeniculate leaflet: an anatomically and functionally distinct subdivision of the lateral geniculate complex. J Comp Neurol 344:403-430.

Moore RY, Lenn NJ (1972) A retinohypothalamic projection in the rat. J Comp Neurol 146:1-14.

Moore RY, Speh JC (1993) GABA is the principal neurotransmitter of the circadian system. Neurosci Lett 150:112-116.

Morin LP (1994) The circadian visual system. Brain Res Rev 67:102-127.

Morin LP, Blanchard JH, Moore RY (1992) Intergeniculate leaflet and suprachiasmatic nucleus organization and connections in the hamster. Vis Neurosci 8:219-230.

Okamura H, Berod A, Julien JF, Geffard M, Kitahama K, Mallet J, Bobillier P (1989) Demonstration of GABAergic cell bodies in the suprachiasmatic nucleus: in situ hybridization of glutamic acid decarboxylase (GAD) mRNA and immunocytochemistry of GAD and GABA. Neurosci Lett 102:131-136.

Pickard GE (1982) The afferent connections of the suprachiasmatic nucleus of the golden hamster with emphasis on the retinohypothalamic projection. J Comp Neurol 211:65-83.

Piggins HD, Stamp JA, Burns J, Rusak B, Semba K (1996) Distribution of pituitary adenylate cyclase-activating polypeptide (PACAP) immunoreactivity in the hypothalamus and extended amygdala of the rat. J Comp Neurol 376:278-294.

Richter CP (1968) Inherent twenty-four hour and lunar clocks of a primate, the squirrel monkey. Commun Behav Biol 1:305-332.

Schwartz WJ, Gross RA, Morton MT (1987) The suprachiasmatic nuclei contain a tetrodotoxin-resistant circadian pacemaker. Proc Natl Acad Sci USA 84:1694-1698.

Shibata S, Watanabe A, Hamada T, Ono M, Watanabe S (1994) $N$-methyl-D-aspartate induces phase shifts in circadian rhythm of neuronal activity of rat SCN in vitro. Am J Physiol 267:R360-R364.

van den Pol AN (1991) Glutamate and aspartate immunoreactivity in hypothalamic presynaptic axons. J Neurosci 11:2087-2101.

van den Pol AN, Tsujimoto KL (1985) Neurotransmitters of the hypothalamic suprachiasmatic nucleus: immunocytochemical analysis of 25 neuronal antigens. Neuroscience 15:1049-1086. 